\title{
Association between primary immunodeficiency and asthma exacerbation in adult asthmatics
}

\author{
So-Hee Lee ${ }^{1}$, Ga-Young Ban², Su-Chin Kim³ ${ }^{3}$ Chang-Gyu Chung ${ }^{4}$, Hyun-Young Lee ${ }^{3}$, Ji-Ho Lee ${ }^{5}$, and \\ Hae-Sim Park ${ }^{1}$
}

\begin{abstract}
${ }^{1}$ Department of Allergy and Clinical Immunology, Ajou University School of Medicine, Suwon; ${ }^{2}$ Department of Pulmonology and Allergy, Hallym University Kangdong Sacred Heart Hospital, Seoul; ${ }^{3}$ Department of Statistics, Clinical Trial Center, Ajou University Medical Center, Suwon; ${ }^{4}$ Division of Allergy \& Clinical Immunology, Department of Internal Medicine, Keimyung University Dongsan Medical Center, Daegu; ${ }^{5}$ Department of Internal Medicine, Yonsei University Wonju College of Medicine, Wonju, Korea
\end{abstract}

Received: November 20, 2018 Revised : February 11, 2019 Accepted: February 18, 2019

\section{Correspondence to}

Hae-Sim Park, M.D.

Department of Allergy and Clinical Immunology, Ajou University School of Medicine, 164 World cup-ro, Yeongtong-gu, Suwon 16499 , Korea

Tel: $+82-31-219-4411$

Fax: +82-31-219-5154

E-mail: hspark@ajou.ac.kr
Background/Aims: Primary immunodeficiency (PID) is a serious comorbid condition in adult asthmatics that have frequent exacerbations, which requires monthly replacement of intravenous immunoglobulin (IVIG). However, the prevalence and clinical significance of PID in adult asthmatics in Korea have not yet been reported. The aim of this study is to assess the prevalence of PID and its association with asthma exacerbation in Korean adult asthmatics.

Methods: A total of 2,866 adult asthmatics were enrolled in this study. The PID group was defined as subjects who had lower levels of immunoglobulin G (IgG)/ $\mathrm{A} / \mathrm{M}$ and/or IgG subclass presenting with recurrent respiratory infections. Serum samples were assayed for total IgG/A/M by immunoturbidimetry, and IgG subclasses by nephelometry.

Results: Of the 2,866 asthmatic patients enrolled, 157 (5.49\%) had PID (classified as the PID group), while those without PID was classified as the non-PID group. IgG subclass deficiency (58\%) is most prevalent, among which $\mathrm{IgG}_{3}$ subclass deficiency was most common (58\%). The relative risk of asthma exacerbation was 1.70 times higher in the PID group compared to the non-PID group (1.696; 95\% confidence interval, 1.284 to $2.239 ; p<0.001$ ); the prevalence of severe asthma was significantly higher in the PID group than in the non-PID group (32.48\% vs. $13.00 \%$, $p<0.001)$. Thirty-five among 157 patients in the PID group d maintained IVIG to prevent asthma exacerbation.

Conclusions: It is suggested that PID, especially $\operatorname{IgG}_{3}$ subclass deficiency, is a significant risk factor for asthma exacerbation. Screening of IgG subclass levels and IVIG replacement should be considered in the management in adult asthmatics.

Keywords: Asthma; Immunologic deficiency syndromes; Prevalence; Asthma exacerbation; Immunoglobulins

\section{INTRODUCTION}

Primary immunodeficiency (PID) is caused by defects of innate and/or adaptive immune systems. PID has a wide clinical spectrum of congenital and acquired forms in the pediatric population [1]; however, the prevalence of adult-onset PID is not common [2]. Although the preva- lence of PID in the general population has been reported as 1:10,000 to 1:1,200 in Western countries, it varies depending on the types of PID and ethnic groups. A previous report demonstrated that the prevalence of PID was 11.25/1,000,000 in a Korean pediatric population [1], while the prevalence of PID in an adult population is not reported in Korea. 
Major clinical features of PID in adults are recurrent infections in various organs, in which recurrent upper and lower respiratory infections, such as pneumonia and sinusitis, are characteristic. Furthermore, these repiratoty infections are known as major triggering factors for asthma exacerbation in adult asthmatics [3]. Recently, we demonstrated that immunoglobulin $\mathrm{G}_{3}\left(\operatorname{IgG}_{3}\right)$ subclass deficiency $\left(\operatorname{IgG}_{3} \mathrm{SCD}\right)$ is the most common type of PID associated with asthma exacerbation in adult asthmatics [4], in which monthly intravenous immunoglobulin (IVIG) therapy was beneficial for preventing asthma exacerbation [5]. We hypothesized that PID is associated with frequent asthma exacerbations and severe asthma in adult asthmatics. The present study aimed to evaluate the prevalence/characteristics of PID patients and to analyze its association with asthma exacerbation in a cohort of adult asthmatics.

\section{METHODS}

\section{Study subjects}

This is a retrospective study to enroll 2,866 adult asthmatics that had visited the Department of Allergy and Clinical Immunology of Ajou University Hospital, Suwon, South Korea, from April 1994 to March 2016 and was approved by the Institutional Review Board of the Ajou University Hospital (IRB No. AJIRB-MED-MDB-17-432). Written informed consent by the patients was waived due to a retrospective nature of our study. A diagnosis of asthma was established by typical clinical symptoms (such as wheezing, dyspnea, and cough), evidence of airway reversibility (defined as forced expiratory volume of 1 second $\left[\mathrm{FEV}_{1}\right]>12 \%$ or $200 \mathrm{~mL}$ from pre-bronchodilator use) and/or airway hyperresponsiveness to methacholine (defined as a methacholine concentration causing $\mathrm{FEV}_{1}$ fall of $\geq 20 \%$ or a methacholine concentration of $<16 \mathrm{mg} / \mathrm{mL}$ ) according to the Global Initiative for Asthma guideline. Severe asthma was defined according to the European Respiratory Society/American Thoracic Society guideline [6]. Asthma exacerbation was defined when subjects who had maintained anti-asthmatic medications and had episodes of symptom worsening requiring systemic corticosteroids (prednisolone equivalent dose of $\geq 15 \mathrm{mg}$ per day for $\geq 3$ consecutive days). Demographic data on the study subjects, including age, sex, previous medications, associated allergic diseases as well as laboratory findings, were collected. Any patients having comorbid conditions and malignancies to affect PID and asthma exacerbation were excluded. Results of skin prick testing and spirometry performed at the initial visit were collected. Atopy was defined as (1) at least one positive result in serum specific IgE or (2) more than 1 positive skin-prick test response to aeroallergens (cats, dogs, house dust mites, trees, grasses, weeds, or fungi). Using the skin prick test, a mean wheal diameter of $\geq 3$ $\mathrm{mm}$ or a mean erythema diameter of $\geq 10 \mathrm{~mm}$ in the absence of any reaction to the negative control was considered to indicate a positive reaction. Total IgE and specific IgE levels in sera were measured by the Immuno CAP system (ThermoFisher Scientific, Waltham, MA, USA). Sputum eosinophil/neutrophil counts were assessed; eosinophilic asthma was defined as study subjects having sputum eosinophilia of $\geq 3 \%$, while neutrophilic asthma was defined as those having sputum neutrophilia of $\geq 65 \%$.

The PID group was defined as a group of asthmatics with recurrent infections who had lower levels of serum IgG/M/A and/or IgG subclasses according to 10 warning signs of Jeffery Modell Foundation [7]. The following patients were excluded from the study: (1) those with secondary immunodeficiency such as human immunodeficiency virus infection, malignancies or other viral infections; and (2) those treated with longstanding systemic steroids and immunosuppressive medications. The non-PID group was defined as a group of patients who had been treated with asthma for more than 2 years and had no PID evidence of clinical or laboratory findings during the study period.

\section{Measurement of serum immunoglobulin levels}

The serum levels of total IgG, IgA, and IgM were measured by the immunoturbidimetric technique (Roche, Marlborough, MA, USA). The reference ranges of normal levels were 916 to $1,796 \mathrm{mg} / \mathrm{dL}$ for IgG, 40 to $260 \mathrm{mg} / \mathrm{dL}$ for IgA, and 93 to $365 \mathrm{mg} / \mathrm{dL}$ for IgM. Four kinds of IgG subclass levels ( $\operatorname{IgG}_{1}$ to $\operatorname{IgG}_{4}$ ) were measured by using nephelometry (BNII, SIEMENS, Newark, DE, USA). Normal ranges of IgG subclass are as follows: 315 to $855 \mathrm{mg} / \mathrm{dL}$ for $\mathrm{IgG}_{1}, 64$ to $495 \mathrm{mg} / \mathrm{dL}$ for $\mathrm{IgG}_{2}, 23$ to $196 \mathrm{mg} / \mathrm{dL}$ for $\mathrm{IgG}_{3}$, and 11 to $157 \mathrm{mg} / \mathrm{dL}$ for $\mathrm{IgG}_{4}$. 


\section{Statistical analysis}

SPSS version 21.0 (IBM Co., Armonk, NY, USA) was used for all statistical analyses. Descriptive statistics for the general patient characteristics were computed for all patients. Continuous variables are expressed as mean \pm standard deviation (SD), and categorical variables are presented as numbers and percentages. To adjust for confounders, logistic regression analysis involving age and sex was conducted to define the effect of PID on clinical characteristics; a generalized estimating

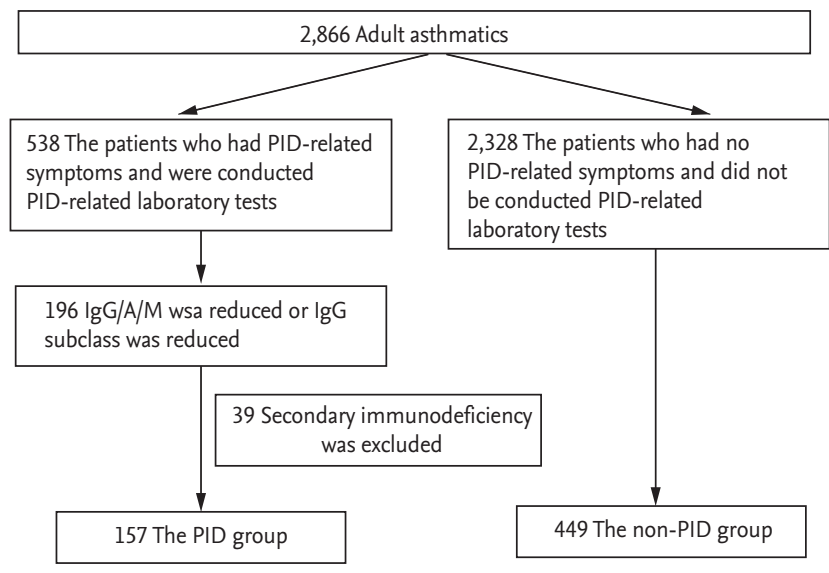

Figure 1. Study design of this study. PID, primary immunodeficiency; IgG/A/M, immunoglobulin G/A/M. equation involving age, sex and severe asthma was used to define the effect of PID on asthma exacerbation; the relative risk (RR), exponential $(\beta)$ and $95 \%$ confidence interval (CI) were calculated. Statistical significance was defined as $p<0.05$ for all analyses.

\section{RESULTS}

\section{Prevalence and distribution of PID}

Fig. 1 shows our study design. Among the 2,866 adult asthmatics screened, laboratory testing for the diagnosis of PID, including IgG/IgA/IgM/IgG subclass levels, were performed on 538 as they had asthma symptoms. Among 196 patients who showed abnormal findings in laboratory testing, 39 were excluded as they were found to have secondary immunodeficiency. Based on our classification criteria for PID, 157 patients were classified as the PID group. The non-PID group included 449 subjects who had neither PID symptoms nor laboratory findings suggestive of PID during the study period. Significant differences were found in age and sex (Table 1), which were adjusted through a logistic regression analysis and a generalized estimating equation. The prevalence of PID in adult asthmatics was found to be $5.49 \%$ ( 157 among the 2,866 subjects). The most common type was IgGSCD

Table 1. Baseline characteristics of the study subjects

\begin{tabular}{|c|c|c|c|}
\hline \multirow{2}{*}{ Clinical parameter } & \multicolumn{2}{|c|}{ Adult asthmatics } & \multirow{2}{*}{$p$ value } \\
\hline & PID group $(n=157)$ & Non-PID group $(\mathrm{n}=449)$ & \\
\hline Age, yr & $47.10 \pm 14.67$ & $41.10 \pm 13.02$ & $<0.001$ \\
\hline Female sex & $115(73.25)$ & $264(58.80)$ & 0.002 \\
\hline Smoking history ${ }^{\mathrm{a}}$ & $33(21.02)$ & $233(51.89)$ & 0.601 \\
\hline Disease period of BA & $6.42 \pm 6.69$ & $6.14 \pm 6.34$ & 0.813 \\
\hline Severe asthma & $51(32.48)$ & $58(13.00)$ & $<0.001$ \\
\hline Total IgE, kU/L & $334.93 \pm 471.26$ & $392.45 \pm 681.46$ & 0.500 \\
\hline Atopy & $29(40.28)$ & $251(57.97)$ & 0.005 \\
\hline $\mathrm{ECP}, \mu \mathrm{g} / \mathrm{L}$ & $34.90 \pm 35.88$ & $33.01 \pm 39.24$ & 0.749 \\
\hline Eosinophilic asthma & $21(13 \cdot 38)$ & $115(25 \cdot 61)$ & 0.713 \\
\hline Neutrophilc asthma & $16(10.19)$ & $114(25 \cdot 39)$ & 0.154 \\
\hline $\mathrm{FEV}_{1}, \%$ pred. & $82.78 \pm 23.49$ & $85.93 \pm 21.65$ & 0.335 \\
\hline
\end{tabular}

Values are presented as mean \pm standard error or number (\%).

PID, primary immunodeficiency; BA, bronchial asthma; IgE, immunoglobulin E; ECP, eosinophil cationic protein; FEV forced expiratory volume in 1 second; pred., predicted.

${ }^{\mathrm{a}}$ Current smoker or ex-smoker. 


\section{A}

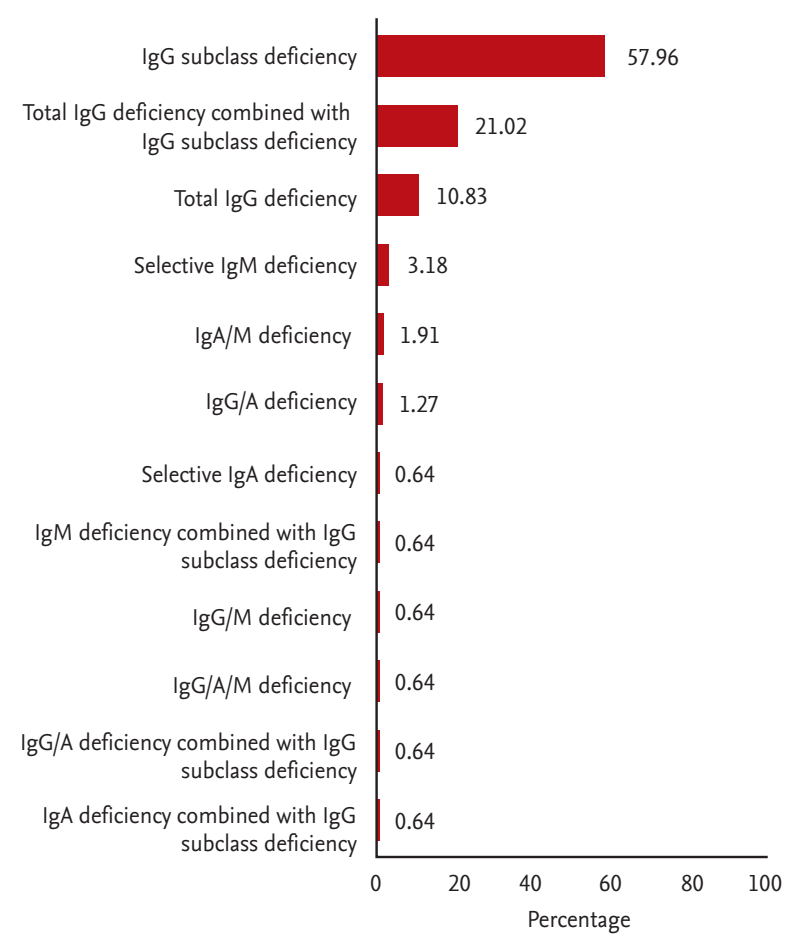

Figure 2. The proportion of primary immunodeficiency among adult asthmatics recruited from a single tertiary center in Korea. (A) Distribution of primary immunodeficiency. (B) Distribution of affected immunoglobulin G/A/M (IgG/A/M) subclass deficiency.

(57.96\%), followed by total IgG plus IgGSCD (21.02\%) and total IgG deficiency (10.83\%) (Fig. 2A). The most common affected IgG subclass was $\operatorname{IgG}_{3}(58.27 \%)$, followed by $\operatorname{IgG}_{4}$ (11.02\%) and $\operatorname{IgG}_{2}$ plus $\operatorname{IgG}_{3}$ (7.09\%) (Fig. 2B).

\section{Comparison of clinical characteristics}

Table 1 shows differences in baseline characteristics between the two study groups. The mean age was older in the PID group than in the non-PID group $(47.10 \pm 14.67$ years vs. $41.10 \pm 13.02$ years, $p<0.001)$. The prevalence of PID was more common in elderly asthmatic patients $(\geq$ 60 years old) than in the nonelderly patients (48.57\% vs. $22.95 \%, p<0.001)$, while the prevalence of PID was significantly lower in younger asthmatics ( $<30$ years old). Negative correlations were found between age and IgG/ $\mathrm{IgG}_{3}$ subclass levels $(r=-0.118, p=0.021$ vs. $r=-0.86, p=$ 0.078 , respectively; data not shown). The number of female patients was significantly larger in the PID group than in the non-PID group (73.25\% vs. $58.80 \%, p=0.002$ ). No differences were observed in smoking history, disease duration, comorbidities, and levels of serum total

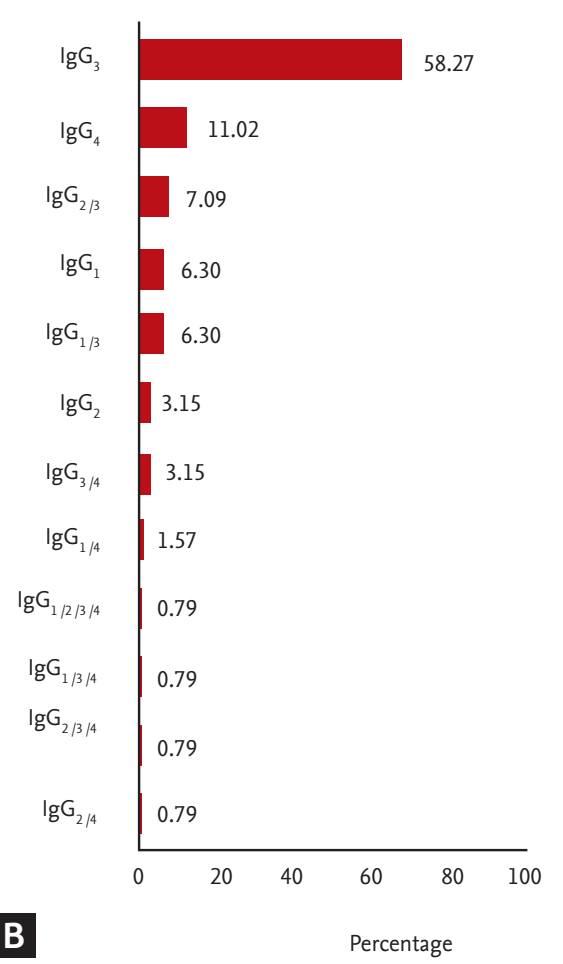

B
IgE and ECP. In addition, there were no differences in $\mathrm{FEV}_{1}$ (\%predicted value) as well as sputum eosinophil/ neutrophil counts between the two groups. However, the prevalence of severe asthma was significantly higher in the PID group than in the non-PID group (32.48\% vs. $13.00 \%, p<0.001)$.

\section{Association between PID and asthma exacerbation}

In the PID group, the RR of asthma exacerbation was 1.70 times higher ( $R R, 1.696$; $95 \%$ CI, 1.284 to 2.239 ; $p<$ 0.001 ), the frequency of asthma exacerbations was significnatly higher in the PID group than in the non-PID group ( $0.68 \pm 1.339$ vs. $0.33 \pm 0.931, p<0.001)$, and the frequency of asthma exacerbation was 1.24 times higher (RR, 1.238; 95\% CI, 1.045 to $1.446 ; p=0.013$ ) than in the non-PID group. In addition, patients with total IgG plus IgGSCD was found to have the highest RR of asthma exacerbation (RR, 1.764; 95\% CI, 1.124 to $2.769 ; p=0.014$ ).

\section{IVIG replacement}

The IVIG maintenance group was defined as a group 
of patients who had been administered more than four times a year, and if treated less than four times a year, they were defined as the IVIG intermittently replaced group. Among 157 asthmatic patients with PID, 50 (31.85\%) were replaced with IVIG more than once. Thirty-five patients (22.29\%) were in the IVIG maintenance group, while 15 (9.55\%) were in the IVIG intermittently replaced group. In the IVIG maintenance group, 27 asthmatics were women and eight asthmatics were men; the mean age starting IVIG replacement was 49.45 \pm 14.78 years (ranging from 24 to 81 years); the mean dose of IVIG replacement was $0.29 \pm 0.15 \mathrm{~g} / \mathrm{kg}$; the mean interval of the IVIG maintenance group was $53.81 \pm 25.41$ days ranging from 23 to 170 days. Among the patients receiving IVIG replacement, $73 \%$ received it in the outpatient clinic, while $27 \%$ was received it in the inpatient ward. One course of IVIG replacement took more than 6 hours $(436.05 \pm 42.68$ minutes) in the outpatient clinic and more than 3 days $(3.32 \pm 3.56$ days $)$ in the inpatient word .The incidence of adverse drug events during the IVIG replacement was found to be $7.66 \%$. The most common reaction was headache, followed by nausea and generalized myalgia $(8.07 \%, 7.34 \%$, and $3.48 \%$, respectively). The average cost of 1 course of IVIG replacement was $590,000 \mathrm{KRW}$, with annual cost ranging from 2,400,000 to $7,200,000 \mathrm{KRW}$.

\section{DISCUSSION}

This is the first study to report the prevalence of PID in an adult asthmatic cohort at a single tertiary hospital setting in Korea and demonstrated a significant association between PID and asthma exacerbation/severe asthma in adult asthmatic patients. Our results suggest that PID, a comorbid condition of adult asthmatics, could increase the RR of asthma exacerbation, which may contribute to the progression to severe asthma.

In the present study, the prevalence of PID in adult asthmatics was higher (5.49\%) than in the general population. Most prevalence studies of PID reported were the overall prevalence in the general population, not in asthmatic cohorts. The prevalence of PID in the general population was reported to range from 0.00077 to 30.5 in 100,000 people according to different regions and ethnic groups: 0.77 to 2.17 per 100,000 in a Taiwanese population [8], 2.3 per 100,000 in a Japanese population [9], 2.7 per 100,000 in a Singaporean population [10], 4.4 per 100,000 in a French population [11], 3.95 per 100,000 in an Argentinean population, 18.61 per 100,000 in a Brazilian population [12], and 30.5 per 100,000 in a Turkish population [13]. To the best of our knowledge, there have been few nationwide surveys of PID in an adult population in Korea, except one study reporting a PID prevalence of 1.13 per 100,000 in a pediatric population [1]. Although the reason the prevalence of PID is higher in adult asthmatics than in the general populations is not fully understood, it is conceivable that asthma is the most common comorbid condition in an adult PID cohort in Korea [4]; asthmatics with PID (especially $\mathrm{IgG}_{3} \mathrm{SCD}$ ) suffered from recurrent respiratory infections, contributing to asthma exacerbation and asthma severity. Therefore, asthmatics with PID can be detected more frequently. In addition, the prevalence of elderly asthmatics is increasing in Korea and worldwide [14-17]. In the present study, the prevalence of PID in adult asthmatics increased in elderly asthmatics, suggesting that PID may be considered a comorbid condition in elderly asthmatic patients who suffer from recurrent respiratory infections and frequent asthma exacerbations. Little is known about changes in serum immunoglobulin levels with age. The relationship between changes in serum immunoglobulin levels and the prevalence of immunological disease are not clarified; however, in the previous study aging is a cause of biological variation indirectly related to immunodeficiency [18] due to decreases in T-cells and switching isotypes of B-cells $[19,20]$. In the case of healthy controls, there is an age-related increase in IgG and IgA, and the researchers suggested that not the quantity but quality is altered with age; however, there were restrictions to functional assays about immunoglobulins [19,21]. Serial changes in serum immunoglobulin levels in PID patients have not yet been evaluated. In the present study, the mean age of the PID group patients was older than that of the nonPID group patients, and there was a negative correlation between age and $\mathrm{IgG} / \mathrm{IgG}_{3}$ levels, suggesting that age may affect the development of PID in adult asthmatic patients. The prevalence of PID was found to be $5.49 \%$ in adult asthmatic patients, indicating that PID can be considered a comorbid condition in the management of severe asthma. 
Our previous study demonstrated that IgGSCD is the most common type of PID in adult asthmatics and patients with IgGSCD have suffered from recurrent/intractable respiratory infections such as rhinosinusitis, otitis media, bronchitis and pneumonia which result in frequent asthma exacerbations [4]. The mechanisms by which IgGSCD, especially $\mathrm{IgG}_{3}$ deficiency, is associated with frequent respiratory infections and asthma exacerbations with poor clinical outcome are not well understood. However, there have been a few studies demonstrating the efficacy of IVIG replacement in asthmatic patients with IgGSCD in whom monthly IVIG replacement significantly improved quality of life, diminished the number of infections and decreased the need for antibiotics and hospitalization $[22,23]$. Our previous multicenter trial demonstrated that IVIG replacement $>6$ months) in adult asthmatics with $\mathrm{IgG}_{3} \mathrm{SCD}$ could significantly reduce the frequency of respiratory infections and asthma exacerbations as well as improve asthma control asthma status and quality of life [5]. Once asthma exacerbation occurs, inflammations and remodeling pathways are activated, which result in the deterioration of lung function. Lung function decline increases the risk of asthma exacerbation and creates a vicious circle between lung function decline and asthma exacerbation. Recurrent respiratory infections, which can permanently reduce lung function, cause asthma exacerbation $[24,25]$. These findings suggest that early detection of PID, including IgGSCD, may be critical for asthmatic patients with frequent asthma exacerbations especially when they are caused by respiratory infections. This indicates that IVIG replacement could prevent asthma exacerbation and lung function decline in adult asthmatics with PID. The IVIG replacement reported standard therapeutic options for most antibody deficiencies such as X-linked agammaglobulinaemia, common variable immunodeficiency, X-linked hyper-IgM, severe combined immunodeficiency, Wiskott-Aldrich syndrome and selective IgG class deficiency [26].

The dose of IVIG administered was recommended as 400 to $600 \mathrm{mg} / \mathrm{kg}$ every 3 to 4 weeks (or the equivalent given in divided doses once or twice a week subcutaneously) $[5,27,28]$. The present study evaluated the current status of IVIG replacement in adult asthmatics in a single tertiary hospital setting and demonstrated that $31.85 \%$ of the PID group patients had maintained
IVIG (probably due to the high cost). Since this study is a retrospective study, it showed inconsistent medication intervals/doses. Further studies are needed to confirm our results.

This study has some limitations. First, this has a retrospective design. Secondly, it has a limitation due to a small sample size done in a single center. Thirdly, we could not measure antibody responses to specific antigen challenges, including pneumococcal vaccine [27]. To evaluate the exact prevalence of PID in adult asthmatics, future research will be needed in multicenter trial settings using the national registry of PID.

In conclusion, our results demonstrated the prevalence of IgGSCD in adult asthmatic patients associated with asthma exacerbation. Early detection and proper management of PID may be critical for the management of adult asthmatics with recurrent asthma exacerbations and severe asthma.

\section{KEY MESSAGE}

1. Primary immunodeficiency (PID), especially immunoglobulin $\mathrm{G}_{3}\left(\operatorname{IgG}_{3}\right)$ subclass deficiency, is a risk factor for asthma exacerbation in adult asthmatics.

2. Early detection and proper management of PID, including intravenous immunoglobulin therapy, may be critical for the management of adult asthmatics with recurrent asthma exacerbations and severe asthma symptoms.

\section{Conflict of interest}

No potential conflict of interest relevant to this article was reported.

\section{Acknowledgments}

This study was supported by Green Cross Corp. and by a grant from the Korean Health Technology R\&D Project, Ministry of Health and Welfare, Republic of Korea (HI16Co992).

\section{REFERENCES}

1. Rhim JW, Kim KH, Kim DS, et al. Prevalence of pri- 
mary immunodeficiency in Korea. J Korean Med Sci 2012;27:788-793.

2. Carneiro-Sampaio M, Moraes-Vasconcelos D, Kokron $\mathrm{CM}$, et al. Primary immunodeficiency diseases in different age groups: a report on 1,008 cases from a single Brazilian reference center. J Clin Immunol 2013;33:716-724.

3. Gathmann B, Mahlaoui N; CEREDIH, et al. Clinical picture and treatment of 2212 patients with common variable immunodeficiency. J Allergy Clin Immunol 2014;134:116-126.

4. Kim JH, Park HJ, Choi GS, et al. Immunoglobulin G subclass deficiency is the major phenotype of primary immunodeficiency in a Korean adult cohort. J Korean Med Sci 2010;25:824-828.

5. Kim JH, Ye YM, Ban GY, et al. Effects of immunoglobulin replacement on asthma exacerbation in adult asthmatics with IgG subclass deficiency. Allergy Asthma Immunol Res 2017;9:526-533.

6. Chung KF, Wenzel SE, Brozek JL, et al. International ERS/ ATS guidelines on definition, evaluation and treatment of severe asthma. Eur Respir J 2014;43:343-373.

7. Modell V, Gee B, Lewis DB, et al. Global study of primary immunodeficiency diseases (PI): diagnosis, treatment, and economic impact: an updated report from the Jeffrey Modell Foundation. Immunol Res 2011;51:61-70.

8. Lee WI, Huang JL, Jaing TH, et al. Distribution, clinical features and treatment in Taiwanese patients with symptomatic primary immunodeficiency diseases (PIDs) in a nationwide population-based study during 1985-2010. Immunobiology 2011;216:1286-1294.

9. Takada H. Primary immunodeficiency in Japan: epidemiology, diagnosis, and pathogenesis. Pediatr Int 2013;55:671674.

10. Lim DL, Thong BY, Ho SY, et al. Primary immunodeficiency diseases in Singapore: the last 11 years. Singapore Med J 2003;44:579-586.

11. Mahlaoui N, Jais JP, Brosselin P, et al. Prevalence of primary immunodeficiencies in France is underestimated. J Allergy Clin Immunol 2017;140:1731-1733.

12. Errante PR, Franco JL, Espinosa-Rosales FJ, Sorensen R, Condino-Neto A. Advances in primary immunodeficiency diseases in Latin America: epidemiology, research, and perspectives. Ann N Y Acad Sci 2012;1250:62-72.

13. Kilic SS, Ozel M, Hafizoglu D, Karaca NE, Aksu G, Kutukculer N. The prevalences [correction] and patient characteristics of primary immunodeficiency diseases in
Turkey: two centers study. J Clin Immunol 2013;33:74-83.

14. Ulambayar B, Lee SH, Yang EM, Ye YM, Park HS. Association between epithelial cytokines and clinical phenotypes of elderly asthma. Allergy Asthma Immunol Res 2019;11:79-89.

15. Ban GY, Ye YM, Kim SH, et al. Plasma LTE4/PGF2 $\alpha$ ratio and blood eosinophil count are increased in elderly asthmatics with previous asthma exacerbation. Allergy Asthma Immunol Res 2017;9:378-382.

16. Eder W, Ege MJ, von Mutius E. The asthma epidemic N Engl J Med 2006;355:2226-2235.

17. Song WJ, Kang MG, Chang YS, Cho SH. Epidemiology of adult asthma in Asia: toward a better understanding. Asia Pac Allergy 2014;4:75-85.

18. Gupta S, Agrawal A, Agrawal S, Su H, Gollapudi S. A paradox of immunodeficiency and inflammation in human aging: lessons learned from apoptosis. Immun Ageing 2006;3:5.

19. Listì F, Candore G, Modica MA, et al. A study of serum immunoglobulin levels in elderly persons that provides new insights into B cell immunosenescence. Ann N Y Acad Sci 2006;1089:487-495.

20. Weksler ME. Changes in the B-cell repertoire with age. Vaccine 2000;18:1624-1628.

21. Radl J, Sepers JM, Skvaril F, Morell A, Hijmans W. Immunoglobulin patterns in humans over 95 years of age. Clin Exp Immunol 1975;22:84-90.

22. Abdou NI, Greenwell CA, Mehta R, Narra M, Hester JD, Halsey JF. Efficacy of intravenous gammaglobulin for immunoglobulin G subclass and/or antibody deficiency in adults. Int Arch Allergy Immunol 2009;149:267-274.

23. Abrahamian F, Agrawal S, Gupta S. Immunological and clinical profile of adult patients with selective immunoglobulin subclass deficiency: response to intravenous immunoglobulin therapy. Clin Exp Immunol 2010;159:344350.

24. Rich AL, Le Jeune IR, McDermott L, Kinnear WJ. Serial lung function tests in primary immune deficiency. Clin Exp Immunol 2008;151:110-113.

25. Verma N, Grimbacher B, Hurst JR. Lung disease in primary antibody deficiency. Lancet Respir Med 2015;3:651660.

26. Kaveri SV, Maddur MS, Hegde P, Lacroix-Desmazes S, Bayry J. Intravenous immunoglobulins in immunodeficiencies: more than mere replacement therapy. Clin Exp Immunol 2011;164:2-5. 
27. Albin S, Cunningham-Rundles C. An update on the use of immunoglobulin for the treatment of immunodeficiency disorders. Immunotherapy 2014;6:1113-1126.

28. Quinti I, Soresina A, Guerra A, et al. Effectiveness of im- munoglobulin replacement therapy on clinical outcome in patients with primary antibody deficiencies: results from a multicenter prospective cohort study. J Clin Immunol 2011;31:315-322. 